\title{
Identification and Characterization of Defensin Genes in Brassica juncea and Camelina sativa and Analysis of their Expression in Response to Biotic and Abiotic Stress
}

\section{Gamage Dona Gaya Chaturani, Zahoor Ahmad Mir, Prashant Mishra and Anita Grover*}

National Institute for Plant Biotechnology, New Delhi, India

*Corresponding Author: Anita Grover, National Institute for Plant Biotechnology, New Delhi, India

Received: October 28, 2019; Published: November 29, 2019

DOI: $10.31080 /$ ASAG.2019.03.0732

\begin{abstract}
Plants are attacked by various biotic and abiotic factors throughout their lives and how efficient and effective the defense mechanisms are, determine the survival. Defensins, belong to Pathogen Related (PR) protein class-12. They are one of the key modulators in plant defense mechanisms which have diverse functions including inhibitory effect on a broad range of phytopathogenic fungi, and are involved in biotic stress response. In depth study on defensins gives an insight into their role in defense which further can be used in conferring disease resistance in crop plants. In-silico analysis identified 56 putative defensins in $C$. sativa (resistant to Alternaria blight) whereas, only six were identified in B. juncea (sueptible toAlternaria blight). Multiple sequence alignment and consensus analysis confirmed the conserved eight cysteine residues, and structural analysis revealed the presence of an $\alpha$-helix and triple strand anti parallel $\beta$-sheets. Defensin genes identified were having a single intron, and identification of Methyle Jasmonate (MeJA) responsive elements in promoter analysis confirm their regulation under Jasmonic Acid (JA) signalling pathway. These defensins were phylogenetically related to other Brassica species as well as to Arabidopsis indicating their close evolutionary relationship. Expression analysis indicated that defensin is responsive to JA, Salicylic Acid (SA), Alternaria infection, and wounding. The antagonistic effect between SA and JA was observed in defensin gene expression in response to their exogenous application. This study will provide an important foundation for further investigation on defensin as a possible source for creating disease resistance in transgenics.
\end{abstract}

Keywords: Defensin; Brassica; Camelina; JA; SA; Alternaria Blight

\section{Introduction}

Alternaria blight of cruciferous vegetables, incited by different species of Alternaria, remains an increasing threat to Brassicaceae crops throughout the world. In oil seed rape, A. brassicae is the dominant invasive species [37]. A. brassicae, is one of the most common and destructive disease of Indian mustard (Brassica juncea) and the yield losses have been estimated to range from 35 to $46 \%$ [32]. Conventional breeding to develop resistant cultivars in $B$. juncea against $A$. brassicae is confounded due to non-availability of suitable resistant sources within the available germplasm of cultivated species of Brassica [35], though some varieties differ in their resistance level. A high degree of resistance to A. brassicae has been found in the wild relatives of Brassica outside the tribe Brassicaceae including Camelina sativa [21]. Thereby, there are necessities of adopting biotechnological strategies to improve resistance against this pathogen. Biotechnological approaches can successfully be utilized to develop resistant variety of $B$. juncea provided that molecular mechanism of defense is delineated.

The molecular mechanisms underlying activation of plant defense responses are exceedingly complex [14] and understanding how plants defend themselves against a range of pathogens is therefore of crucial importance for successful agriculture [1]. Once plant defense responses are activated at the site of infection, a systemic defense is often triggered in distal plant parts to protect these undamaged tissues against subsequent invasion by the pathogen [40]. Systemic defense is controlled by a complex signal transduction network [44] that coordinate the overall defense responses of the plant [19]. Resistance to biotrophic pathogens is 
mediated through phyto-hormones such as salicylic acid (SA) [40] and necrotrophic pathogens by jasmonic acid (JA) and ethylene (ET)-signaling pathways [28].

Various novel proteins are induced [29] collectively known as PR proteins [09] is a group of the most important inducible defense-related antifungal proteins [33]. They are associated with the development of systemic acquired resistance in wide range from cell wall rigidification to signal transduction and antimicrobial activity [26]. They are the key modulators of plant defense [13] which cause activation of a different set of defence related genes, including plant defensin genes [44]. Plant defensins which belong to PR-12 family of PR proteins, are cysteine rich, highly basic [50] prominent cationic peptide in plants [03] with different roles in defense [25, 39]. They are one of the largest families of antifungal peptide molecules [10]. Since the beginning of 1990s, many cationic plant cysteine rich antimicrobial peptides have been studied and plant defensins were first described in the seeds of wheat and barley $[05,31]$.

The main biological function of plant defensins was found to inhibit the growth of broad range of phyto-pathogenic fungi at micro-molecular concentrations. The precise mechanism of action that is employed by plant defensins to inhibit the fungi is not completely understood, although it is generally accepted that they act at the level of the plasma membrane [23]. While some results point to cellular membrane as the point of action, others suggest intracellular targets [49]. There are two major hypothesis which explain the mechanism of action of antimicrobial defensin: the carpet model and the pore model. In both models, defensins are described to interact with the negatively charged molecules present at the cell membrane of pathogens, causing an increase of its permiabilization, leading to cell leakage and death by necrosis [22]. In addition to being antimicrobial, plant defensins are also involved in the biotic stress response, as well as plant growth and development [48].

In Brassica only a few defence related genes have been reported. These include PR proteins including few plant defensins [15], $\beta$-glucanases [36], and Chitinases [55]. Tiwari [53] studied the effect of methyl jasmonate on disease severity and expression of plant defensin gene during A. brassicae infection in Arabidopsis and he has reported that transcripts of PDF1.2 accumulated at a greater level upon challenge inoculation with $A$. brassicae along with jasmonic acid compared to treatment containing pathogen as well as jasmonic acid alone. However no attempts have been undertaken to find the number of defensin genes present in $B$. juncea or in Alternaria resistant $C$. sativa and the structural and functional characteristics of defensins from above two genotypes remain to be unclear. Rawat [41] isolated the full-length pathogen-inducible plant defensin gene from $B$. juncea and have reported its similarity with gamma thionin and knottin families of plant antimicrobial peptides. Efforts are being made in order to have sufficient knowledge about the genes induced during infection and their regulation measures. Therefore the objective of this study is to characterize different defensin genes in $B$. juncea, $C$. sativa and related species to find their structure, evolution, cellular localization, and studying their regulation in response to SA, JA and Alternaria infection.

\section{Materials and Methods}

Genome wide identification of defensin genes in $B$. juncea and C. sativa

The available Arabidopsis thaliana defensin genes were downloaded from TAIR database (https://www.arabidopsis.org/), and their coding sequences were identified using ORFfinder program (https://www.ncbi.nlm.nih.gov/orffinder/). By using the ExPASy translate tool (https://web.expasy.org/translate/) the coding sequences were translated to peptide sequences. The above peptide sequences of $A$. thaliana defensin genes were queried against $B$. juncea and $C$. sativa peptide sequences in BRAD database (http:// brassicadb.org/brad/) to find putative defensins in B. juncea genome using blastp. From the blast output the peptide sequences showing >100 bit score were selected and redundant hits were removed to select unique sequences.

Protein structure, conserved domain identification and computation of physical and chemical parameters

The NCBI-CDD database (https://www.ncbi.nlm.nih.gov/Structure/cdd/wrpsb.cgi) was used to analyze the conserved domain of all non-redundant sequence and SMART (http://smart.embl-heidelberg.de/) and Pfam (https://pfam.xfam.org/) were used to confirm that identified genes were members of defensin family. Those protein containing gamma-thionin domain under Knot1 superfamily were defined to be belonging to the defensin family (previously known as gamma-thionin family). Full length amino acid sequences of the selected defensin proteins were compiled and aligned using ClustalW and the alignment was visualized by Bio Edit V 7.0.4.software to find conserved regions, and the three dimensional secondary structure of defensin proteins were constructed by a ProMod3 Version 1.3.0. homology modelling method using SWISS-MODEL server (https://swissmodel.expasy.org/interactive) to confirm that the selected proteins have stuctures similar to plant defensins, as they are generally defined by their conserved cysteine scaffold with $\alpha$-helix and triple strand anti parallel $\beta$-sheets connected to the scaffold. Computer analysis of the amino acid sequence to com- 
pute chemical and physical parameters were performed with the ProtParam tool on the ExPASy server (https://web.expasy.org/ protparam/) and possible disulphide bridges were determined using the DISUFIND server (http://disulfind.dsi.unifi.it/). The subcellular localization of each defensin protein of $B$. juncea and C. sativa was predicted using Plant-mPLoc (http://www.csbio.sjtu. edu.cn/bioinf/plant-multi/).

Gene Structure construction and prediction of cis-acting elements

The defensin sequences retrieved from BRAD database were employed to identify respective genes from $B$. juncea and $C$. sativa genome using local blast in Bio Edit V 7.0.4.software. The exonintron structure of $B$. juncea defensin encoding genes were determined based on alignment of coding sequences with corresponding genome sequences and graphical display was created using online Gene Structure Display Server (http://gsds.cbi.pku.edu. $\mathrm{cn} /$ ). Conserved cis-acting regulatory elements in the promoter region of the putative defensin genes were analyzed by plant care database (http://bioinformatics.psb.ugent.be/webtools/plantcare/html/).

Phylogenetic analysis of defensin proteins of $B$. juncea, $C$. sativa and related species

To compare the composition of the defensin gene family in Brassica, defensin genes were identified in three other Brassica species, B. rapa, B. napus and B. oleracea and also the wild type $C$. sativa (http://brassicadb.org/brad/) using the same method. To study the evolutionary relationships among B. juncea, B. rapa, $B$. oleracea B. napus and $A$. thaliana defensins, an unrooted neighborjoining phylogenetic tree was constructed using MEGA 7.0 software based on the amino acid sequences of the defensin proteins of above genotype and a bootstrap test with 1000 replicates was performed.

Expression analysis of defensin genes in B. juncea and $C$. sativa in response to $\mathrm{SA}$, JA and $A$. brassicae infection and wounding

B. juncea and $C$. sativa plants were raised from seeds in pots and were maintained at $25^{\circ} \mathrm{C}$ for $16 \mathrm{~h}$ light $/ 8 \mathrm{~h}$ dark in glass house at National Phytotron Facility, Indian Agriculture Research Institute (IARI). A. brassicae isolated from disease infected field grown plants was cultured on Radish Dextrose Agar medium and was identified by Indian Type Culture Collection, IARI (ID No. 81651). Conidial suspensions were prepared by scraping sporulated mycelium from 21-day old cultures and suspending in sterilized distilled water. Conidial concentration was adjusted to $5 \times 10^{3}$ conidia $/ \mathrm{ml}$ using hemocytometer. 45 days old healthy plants with six leaves, were treated with conidial suspensions using a hypodermic needle and control plants were treated same way with sterilized distilled water. To study the effect of hormone on defensin gene expression, plants were sprayed with $100 \mu \mathrm{M}$ MeJA, and $2 \mathrm{mM}$ SA solutions separately and control plants were sprayed with sterilized distilled water. Small puncture wounds were made on the leaves using a hypodermic needle filled with sterilized distilled water, to analyze the effect of wounding on the expression of defensin genes. Leaf samples were collected at $3,6,12,24,48,72$ and $96 \mathrm{~h}$ post treatment. Total RNA was isolated from leaf tissues using TRIzol reagent (Invitrogen $^{\mathrm{TM}}$ ) and first strand cDNA synthesis was performed from 2 $\mu \mathrm{g}$ total RNA using Superscript III cDNA synthesis kit (Invitrogen ${ }^{\mathrm{TM}}$ ) following the manufacturer's instructions. Quantitative real time PCR was performed using SYBR Premix Ex Taq Kit (Takara ${ }^{\mathrm{TM}}$ ) run at $95{ }^{\circ} \mathrm{C}$ for $10 \mathrm{~min}$ followed by 40 cycles of $95^{\circ} \mathrm{C}$ for $10 \mathrm{~s}$ and $55^{\circ} \mathrm{C}$ for $30 \mathrm{~s}$ with F-5' CATGAAGCTCTCTATGCG 3'and R-5' CGATGGATCAGCGATTTCTGG 3' primers. $\beta$-tubulin was used as the reference gene for mRNA relative expression pattern analysis. The reactions were performed with three biological replicates and three technical replicates per sample. The relative quantification method $(\Delta \Delta \mathrm{CT})$ was used to evaluate quantitative variation between the samples.

\section{Results and Discussion}

Genome wide identification of defensin genes in $B$. juncea and C. sativa

During in-silico analysis, fifteen defensin protein coding sequences were identified in A. thaliana from the TAIR database. They were sub divided into three sub families, which seven belonging to family I, six to family II and two plant defensins in family III. All the coding sequences were converted into their respective amino acid sequences and used as a reference to find the plant defensins in $B$. juncea, B. rapa B. oleracea, B. napus and C. sativa. Availability of $B$. juncea genome sequence made it possible to identify all the defensin proteins present in Brassica. When $B$. juncea genome was queried against 15 defensin proteins of $A$. thaliana as described above, only six putative defensin proteins namely BjuB040113, BjuB040109, BjuB027448, BjuA030410 BjuA025766 and BjuB040111 were identified by the analysis that matches to the criteria of plant defensins. However comparative to low number of defensins in $B$. juncea, 56 defensin proteins were identified in $C$. sativa belonging to plant defensins. Twelve defensins were identified both in $B$. rapa and B. oleracea. Surprisingly no defensins were identified in $B$. nigra. Since $B$. juncea was evolved from cross between $B$. nigra and $B$. rapa, identification of only six defensins in $B$. juncea can be justified. However total 42 of defensin proteins were identified in $B$. napus indicating the possibility of gene duplications. 
Analysis of physical, chemical and structural properties of defensin proteins

Computer analysis of physical and chemical parameters of all the defensin proteins was done by ProtParam (Table 1). The length of the defensin proteins ranged from 77 to 82 amino acid residues in B. juncea while in C. sativa it ranged from 72 to 131 amino acid residues. Relative molecular mass varied from $7.79 \mathrm{kDa}$ to 14.33 $\mathrm{kDa}$, while the theoretical iso-electric point measurement varied from 4.81 to 12.00 . Instability index of all the defensin proteins identified was also calculated. A protein whose instability index is smaller than 40 is predicted as stable; a value above 40 predicts that the protein may be unstable. Instability index indicates that four $B$. juncea defensin proteins out of six proteins were unstable while most defensins in $A$. thaliana and $C$. sativa were stable. Instability index provides an estimate of the stability of the protein in a test tube. Calculations on aliphatic index indicated that it varies with 32.52 to 91.17 in all three genotypes. Grand average of hydropathy (GRAVY) calculations indicted that most of the defensins are polar. GRAVY is an indication of hydropathy values of amino acids in a protein; negative values indicates that the protein is non-polar and positive values indicates that the protein is polar.

\begin{tabular}{|c|c|c|c|c|c|c|c|c|}
\hline Prot. ID & $\begin{array}{c}\text { Number } \\
\text { of aa }\end{array}$ & $\begin{array}{c}\text { Molecular } \\
\text { Weight (kDa) }\end{array}$ & $\begin{array}{c}\text { Theoritial } \\
\text { pI }\end{array}$ & $\begin{array}{l}\text { Instability } \\
\text { index }\end{array}$ & $\begin{array}{c}\text { Aliphatic } \\
\text { index }\end{array}$ & $\begin{array}{l}\text { Number of } \\
\text {-ve residue }\end{array}$ & $\begin{array}{l}\text { Number of } \\
\text { +ve residue }\end{array}$ & GRAVY \\
\hline NM_106233.4 & 80 & 8.709 & 8.47 & 27.49 & 74.50 & 4 & 7 & 0.339 \\
\hline NM_123809.4 & 80 & 8.518 & 8.14 & 27.68 & 81.88 & 4 & 6 & 0.454 \\
\hline NM_128161.2 & 80 & 8.640 & 8.14 & 28.23 & 78.12 & 4 & 6 & 0.389 \\
\hline NM_123810.3 & 80 & 8.550 & 8.14 & 25.27 & 75.75 & 4 & 6 & 0.358 \\
\hline NM_128160.3 & 80 & 8.580 & 8.14 & 25.28 & 78.25 & 4 & 6 & 0.436 \\
\hline NM_101817.4 & 78 & 8.840 & 8.44 & 55.88 & 52.69 & 5 & 8 & 0.113 \\
\hline NM_104375.2 & 80 & 9.139 & 5.65 & 31.07 & 65.88 & 9 & 8 & 0.006 \\
\hline NM_126272.4 & 77 & 8.056 & 9.02 & 28.58 & 91.17 & 5 & 8 & 0.600 \\
\hline NM_126271.3 & 77 & 8.524 & 9.37 & 40.13 & 63.25 & 3 & 10 & 0.201 \\
\hline NM_126273.4 & 77 & 8.544 & 9.63 & 39.18 & 59.48 & 3 & 12 & 0.004 \\
\hline NM_104788.4 & 76 & 8.289 & 8.52 & 51.47 & 80.79 & 5 & 8 & 0.182 \\
\hline NM_125761.2 & 73 & 8.387 & 8.92 & 56.73 & 54.79 & 4 & 9 & -0.130 \\
\hline NM_126274.3 & 73 & 7.718 & 8.92 & 76.05 & 73.56 & 3 & 8 & 0.281 \\
\hline NM_123194.3 & 122 & 13.122 & 6.05 & 31.71 & 67.95 & 12 & 11 & -0.130 \\
\hline NM_119153.2 & 129 & 13.862 & 5.57 & 43.91 & 55.89 & 12 & 10 & -0.154 \\
\hline BjuB040113 & 80 & 8.734 & 8.47 & 30.55 & 78.25 & 4 & 7 & 0.335 \\
\hline BjuB040109 & 80 & 8.862 & 9.14 & 29.92 & 68.50 & 5 & 12 & 0.101 \\
\hline BjuB027448 & 77 & 8.574 & 9.33 & 45.54 & 58.18 & 4 & 11 & 0.031 \\
\hline BjuA030410 & 78 & 8.814 & 8.85 & 57.46 & 60.13 & 4 & 9 & 0.103 \\
\hline BjuA025766 & 83 & 8.592 & 12.00 & 89.44 & 64.34 & 6 & 13 & 0.200 \\
\hline BjuB040111 & 82 & 8.905 & 6.24 & 63.66 & 68.05 & 8 & 7 & 0.170 \\
\hline Csa07g047380.1 & 80 & 8.734 & 8.47 & 26.02 & 74.50 & 4 & 7 & 0.225 \\
\hline Csa09g080580.1 & 80 & 8.691 & 8.47 & 26.00 & 75.75 & 4 & 7 & 0.291 \\
\hline Csa16g039940.1 & 80 & 8.744 & 8.47 & 27.48 & 74.50 & 4 & 7 & 0.215 \\
\hline Csa16g039930.1 & 80 & 8.619 & 8.15 & 31.90 & 77.00 & 4 & 6 & 0.310 \\
\hline Csa13g034620.1 & 80 & 8.665 & 8.15 & 29.77 & 75.67 & 4 & 6 & 0.351 \\
\hline Csa09g080550.1 & 80 & 8.707 & 8.16 & 32.96 & 77.01 & 4 & 6 & 0.348 \\
\hline Csa09g080530.1 & 80 & 8.676 & 8.48 & 28.94 & 73.38 & 4 & 7 & 0.201 \\
\hline Csa09g080540.1 & 80 & 8.709 & 8.15 & 31.90 & 75.74 & 4 & 6 & 0.324 \\
\hline Csa09g080520.1 & 80 & 8.736 & 8.48 & 28.94 & 73.99 & 4 & 7 & 0.246 \\
\hline
\end{tabular}




\begin{tabular}{|c|c|c|c|c|c|c|c|c|}
\hline Csa09g080500.1 & 80 & 8.799 & 8.48 & 29.13 & 75.01 & 4 & 6 & 0.309 \\
\hline Csa16g039910.1 & 80 & 8.798 & 8.47 & 29.11 & 75.77 & 3 & 6 & 0.308 \\
\hline Csa16g039920.1 & 80 & 8.742 & 8.87 & 25.59 & 68.50 & 4 & 7 & 0.241 \\
\hline Csa09g080480.1 & 80 & 8.792 & 8.87 & 22.87 & 78.00 & 3 & 8 & 0.143 \\
\hline Csa07g047330.1 & 80 & 8.790 & 8.14 & 23.93 & 80.50 & 3 & 8 & 0.179 \\
\hline Csa05g073650.1 & 80 & 8.846 & 6.00 & 24.05 & 64.75 & 5 & 4 & 0.325 \\
\hline Csa05g073660.1 & 80 & 8.831 & 6.00 & 24.05 & 69.62 & 5 & 4 & 0416 \\
\hline Csa16g039870.1 & 80 & 8.806 & 8.87 & 23.93 & 85.38 & 3 & 8 & 0.246 \\
\hline Csa11g046460.1 & 79 & 8.782 & 8.42 & 33.48 & 65.57 & 4 & 7 & 0.161 \\
\hline Csa07g047360.1 & 75 & 8.195 & 8.36 & 28.85 & 86.00 & 4 & 6 & 0.281 \\
\hline Csa12g074210.1 & 78 & 8.693 & 8.42 & 33.78 & 71.41 & 4 & 7 & 0.238 \\
\hline Csa09g080510.1 & 80 & 8.792 & 8.16 & 33.11 & 60.00 & 4 & 6 & 0.233 \\
\hline Csa07g047340.1 & 82 & 9.064 & 8.16 & 35.51 & 80.98 & 5 & 7 & 0.580 \\
\hline Csa16g039900.1 & 82 & 8.873 & 8.93 & 30.00 & 73.90 & 3 & 8 & 0.556 \\
\hline Csa16g039890.1 & 82 & 8.961 & 8.49 & 33.50 & 94.02 & 5 & 8 & 0.657 \\
\hline Csa07g047350.1 & 82 & 9.022 & 8.72 & 27.38 & 86.95 & 5 & 9 & 0.570 \\
\hline Csa03g023400.1 & 78 & 8.844 & 8.45 & 55.04 & 51.41 & 5 & 8 & 0.047 \\
\hline Csa17g025410.1 & 78 & 8.695 & 8.12 & 45.86 & 51.12 & 5 & 7 & 0.131 \\
\hline Csa11g002160.1 & 78 & 8.934 & 8.77 & 35.63 & 65.13 & 5 & 10 & -0.158 \\
\hline Csa04g007620.1 & 77 & 8.671 & 10.04 & 42.45 & 56.88 & 3 & 13 & -0.044 \\
\hline Csa06g002180.1 & 77 & 8.721 & 10.00 & 42.55 & 58.88 & 3 & 13 & -0.053 \\
\hline Csa06g002170.1 & 77 & 8.694 & 9.69 & 37.13 & 61.95 & 4 & 13 & -0.023 \\
\hline Csa04g007610.1 & 77 & 8.720 & 9.49 & 41.86 & 61.90 & 4 & 12 & 0.006 \\
\hline Csa00456s080.1 & 112 & 12.583 & 9.77 & 41.80 & 63.48 & 8 & 19 & -0.157 \\
\hline Csa16g052960.1 & 74 & 7.998 & 7.52 & 49.61 & 81.62 & 5 & 6 & 0.408 \\
\hline Csa00456s090.1 & 73 & 8.240 & 8.96 & 69.01 & 68.08 & 5 & 10 & 0.047 \\
\hline Csa18g037270.1 & 72 & 8.435 & 8.74 & 62.68 & 55.56 & 5 & 9 & -0.133 \\
\hline Csa06g002200.1 & 73 & 7.792 & 8.92 & 54.62 & 73.56 & 3 & 8 & 0.281 \\
\hline Csa04g007630.1 & 73 & 7.802 & 8.92 & 59.89 & 73.56 & 3 & 8 & 0.270 \\
\hline Csa00456s050.1 & 73 & 7.823 & 8.91 & 54.62 & 73.50 & 3 & 8 & 0.255 \\
\hline Csa16g045530.1 & 122 & 12.937 & 8.26 & 29.81 & 58.36 & 8 & 12 & -0.053 \\
\hline Csa10g044380.1 & 130 & 14.004 & 7.79 & 41.00 & 58.38 & 9 & 11 & -0.060 \\
\hline Csa12g017170.1 & 131 & 13.978 & 8.09 & 36.48 & 57.33 & 8 & 11 & -0.145 \\
\hline Csa11g013400.1 & 131 & 14.083 & 7.81 & 39.82 & 58.78 & 10 & 12 & -0.147 \\
\hline Csa12g084420.1 & 120 & 12.842 & 7.31 & 57.62 & 61.67 & 10 & 11 & -0.072 \\
\hline Csa20g039330.1 & 124 & 13.490 & 6.65 & 22.24 & 73.87 & 13 & 13 & 0.095 \\
\hline Csa12g017150.1 & 130 & 14.169 & 5.65 & 26.82 & 57.08 & 14 & 12 & -0.204 \\
\hline Csa10g012480.1 & 130 & 14.335 & 6.67 & 33.24 & 36.77 & 14 & 14 & -0.376 \\
\hline Csa11g073450.1 & 113 & 12.351 & 8.48 & 18.48 & 80.27 & 9 & 14 & 0.245 \\
\hline Csa11g013370.1 & 111 & 12.098 & 4.81 & 42.21 & 32.52 & 16 & 10 & -0.601 \\
\hline Csa12g085440.1 & 114 & 12.231 & 8.28 & 56.83 & 66.67 & 5 & 8 & -0.112 \\
\hline Csa17g089850.1 & 123 & 13.264 & 5.21 & 55.25 & 68.21 & 10 & 9 & 0.004 \\
\hline
\end{tabular}

Table 1: Chemical and physical parameters of defensin proteins in A. thaliana, B. juncea and C. sativa. 
Controlling the stability of cellular proteins is a fundamental way by which cells regulate growth, differentiation, survival, and development. Measuring the turnover rate of a protein is often the first step in assessing whether or not the function of a protein is regulated by proteolysis under specific physiological conditions [56]. The half-life is a prediction of the time it takes for half of the amount of protein in a cell to disappear after its synthesis in the cell. ProtParam estimates the half-life by looking at the $\mathrm{N}$-terminal amino acid of the sequence under investigation [12] and it was estimated that defensin proteins have more than $10 \mathrm{hrs}$ of half-life in E-coli.

The subcellular localization prediction revealed that these proteins exhibited cell membrane and vacuolar localization. According to Donnes and Hoglund [8], the organelles present unique biological conditions to the proteins. It has been observed that proteins from different organelles differ in their overall amino acid composition and each protein has evolved over time to function optimally in a certain subcellular localization. Plant defensins seem to have specific activities depending on whether they are over-expressed by plant tissues or applied externally [38]. The subcellular localization of plant defensins thus appears to be a crucial point to understand their dual action on plant cells. As all plant defensins have a strongly hydrophobic $\mathrm{N}$-terminal sequence that may serve as a signal peptide and have no obvious internal retention signal, they are generally predicted to be secreted proteins. However, most of the $B$. juncea plant defensins are predicted to be localized in vacuole. The same was observed [24] in NaD1 in Nicotiana alata flowers. However, their internal location is not incompatible with their antimicrobial activity as Sticher., et al. [46] confirmed that when cells are damaged, defensins are released from internal stores to protect tissues against fungal pathogens.

The defensin proteins are characterized by specific sequence diversity and structural feature homology. The protein domains of these defensin proteins were identified and similar protein structure and domain compositions were found, demonstrating that the protein structure is remarkably conserved within the plant defensins. All non-redundant sequence were used to confirm that identified proteins of $B$. juncea were members of defensin family (Figure 1). Those proteins containing gamma-thionin domain under Knot1 superfamily were defined to be belonging to the defensin family [previously known as gamma-thionin family]. Defensin and defensin like peptides are functionally diverse namely disrupting microbial membranes and acting as ligands for cellular recognition and signalling. According to Stotz., et al. [47], the first members of the family of plant defensins were isolated from wheat and barley grains in the early 1990s and those proteins were originally called gamma-thionins because of their size and cysteine content that were found to be similar to thionins. Subsequently gamma-thionins homologous proteins were identified and it as noticed that the plant peptides' structural and functional properties resemble those of insect and mammalian defensins, and therefore later termed the family of peptides 'plant defensins' in. Gachomo., et al. [11] also reported that the plant defensins are structurally similar to their insect counterparts despite the low amino acid sequence similarity between these two organisms. Maarof and co-workers [30] also reported the presence of Knot1 domain and gamma-thionin domain in their studies on CDef1 in Capsicum. The Knot1 domain or knottins represent plant antimicrobial peptides, plant amylase inhibitors, plant gamma-thionins and arthropod defensin.

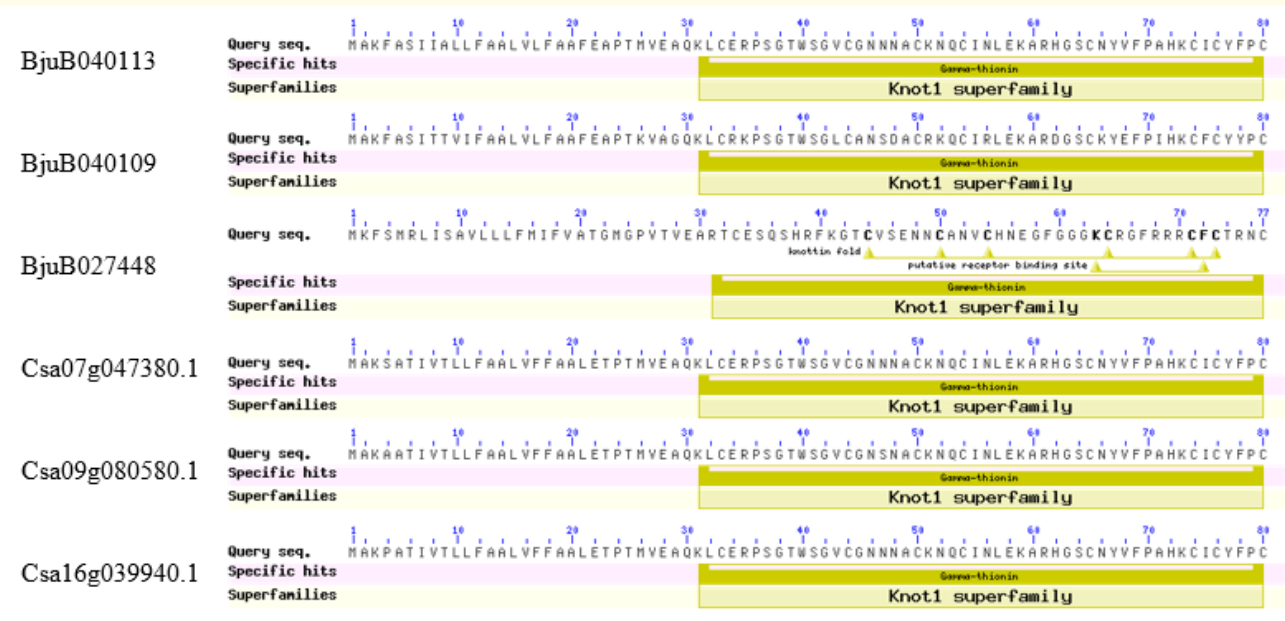

Figure 1: The presence of gamma-thionin domain under Knot1 superfamily in, B. juncea and C. sativa defensins. 
In addition to the conserved domains, other conserved motifs could also be important for the function of plant defensins. Plant defensins share a common motif consisting of an $\alpha$-helix and triple strand anti parallel $\beta$-sheets, a feature that is also shared by several toxins from insects, scorpions, honeybees and spider venom [11]. The structural features of above defensins were analyzed in order to understand the functional characteristics of defensin family. Similar to the domain distribution results, the modeled defensins also share similarity with each other in terms of secondary struc- ture as all the defensin proteins were represented by an $\alpha$-helix and triple strand anti parallel $\beta$-sheets (Figure 2). Despite their structural similarity, plant defensins have great diversity in amino acid sequence. This variation in the primary sequence is associated with the specificity and diverse biological activities of antimicrobial peptides such as antibacterial, antifungal, antioxidant and other various antimicrobial activities [23]. Kaewklom., et al. [18] reported that recently, a conserved ${ }^{\gamma}$-core motif composed of two antiparallel $\beta$-sheets and an interposed loop has been identified and shown to be important for their functions.

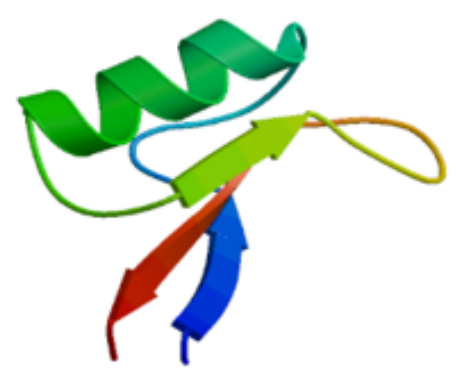

BjuB040113

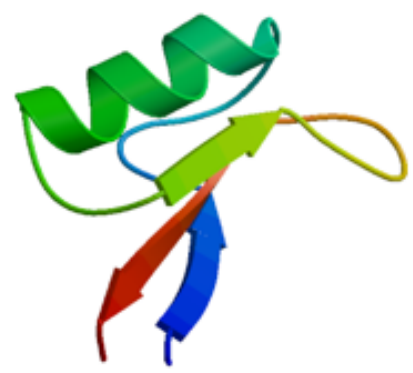

Csa07g047380.1

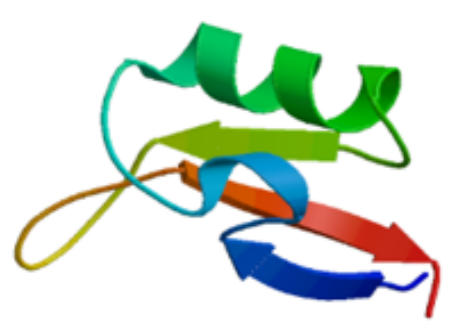

BjuB040109

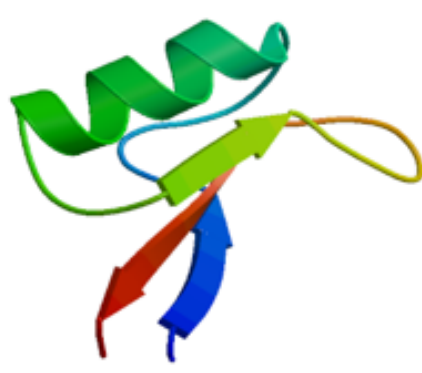

Csa09g080580.1

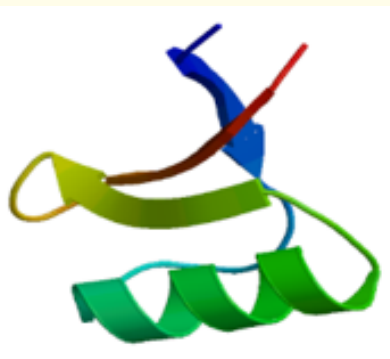

BjuB027448

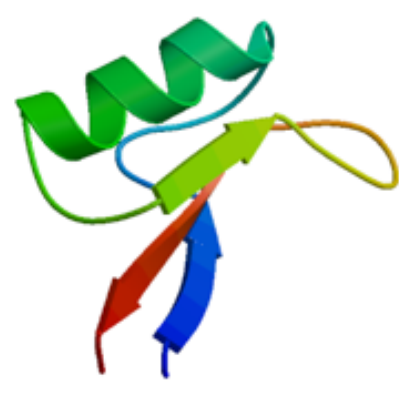

Csa16g039940.1

Figure 2: Secondary structure analysis of $B$. juncea and $C$. sativa defensins.

Multiple sequence alignment of $B$. juncea and $C$. sativa defensin proteins revealed a highly conserved eight cysteine residues near the N-terminal domain of the proteins (Figure 3 and Figure 4). This observation is consistent with the finding of Lay., et al. [23], who suggested that the conservation of these residues may be due to their roles in providing stability and in the folding mechanism, especially the cysteines involved in the formation of disulfide bri- dges. The amino acid sequence conservation of defensin proteins is limited to a small number of residues. These conserved residues are absolutely restricted to the eight cysteines, a glycine residue, as well as a serine and an aromatic residue $(\mathrm{F} / \mathrm{W} / \mathrm{H} / \mathrm{Y})$ that is always followed by another glycine [11]. They are the most conserved positions and essential for structural folding and stabilization of the proteins. 
BjuB0 00113

BjuB0 40109

BjuB027448

BjuA030410

BjuA025766

BjuB040111

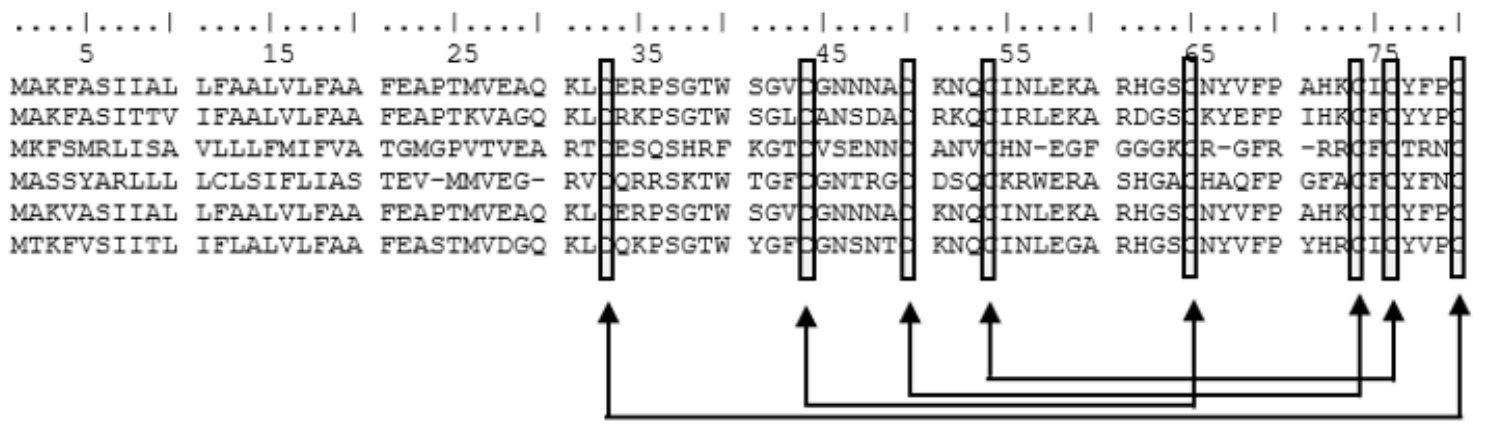

Figure 3: Sequence comparison between plant defensin proteins in B. juncea. The invariable cysteine residues are depicted in gray background. The disulfide bond connectivity is shown below by connecting line.

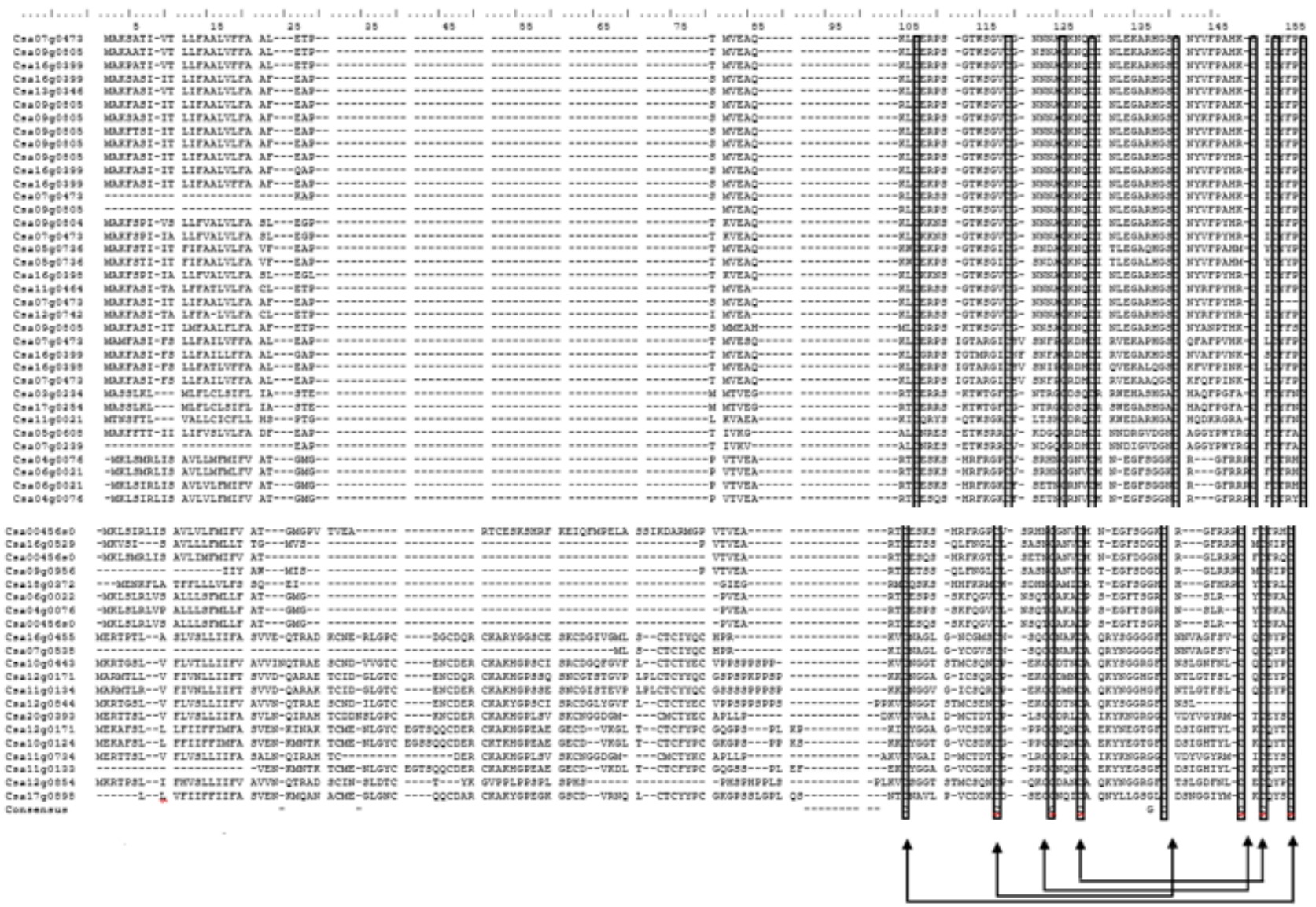

Figure 4: Sequence comparison between plant defensin proteins in C. sativa. 
The overall primary action of plant defensins is to form pores on the membrane of target pathogens and increase membrane permeability [52]. They can resist temperatures near the boiling point without losing function and their structures are well maintained. Their ultra-stability is generally due to the cysteine scaffold of their structures [11]. Conserved amino acid residue analysis of other Brassica members revealed that only eight cysteine residues are strictly conserved among members of the Brassica family. It was reported that the significance of both basic residues and their asymmetric distribution over the molecular surface causes the amphiphilic properties of defensins. Hoover., et al. [17] reported the importance of basic residues for the antimicrobial activity. According to Sagehashi., et al. [42], the site directed mutational analysis of Rs-AFP2, a defensin isolated from radish seeds showed that basic amino acid residues contribute to the antifungal potency of this peptide. The substitutions of neutral residues Gly or Val by arginine increased the Rs-AFP2 antifungal activity. However, according to Lay and Anderson [23], comparison of a series of sequences of plant defensins from various plant species reveals that the plant defensin family shows very limited sequence conservation except for eight cysteine, two glycine and one glutamate acid residues.
Gene Structure construction and prediction of cis-acting elements

To gain further insight into the structural evolution of the defensin genes in $B$. juncea, the exon-intron organization was analyzed by comparing the corresponding genomic sequences with their coding sequences through Gene Structure Display software. Based on the predicted schematic structures, all the genes displayed that a single intron and two exons existed in all the coding sequences (Figure 5). This conserved exon number among all plant defensins in $B$. juncea revealed their close evolutionary relationship. As described by Heyn., et al. [16], the overall rate of transcript production is determined by transcription and RNA processing rates. Gene architectural features, primarily the number and length of exons and introns have recently emerged as important regulatory players. Several new studies indicated that rapidly cycling cells contain gene-architecture toward short genes with few introns, allowing efficient expression during short cell cycles and in genes that need rapid regulation during stress. Therefore, the conserved trend toward shorter gene with fewer introns in plant defensins indicates their rapid induction following pathogen attack.

BjuBO40113

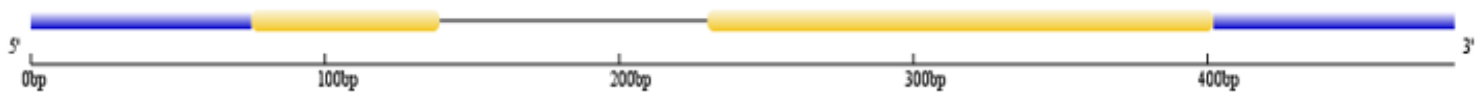

BjuBO40109

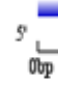

BjuBO27448

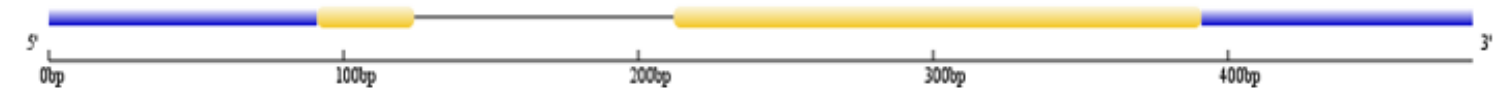

BjuA030410

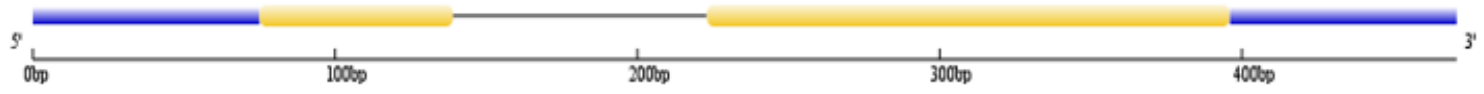

BjuA.025766

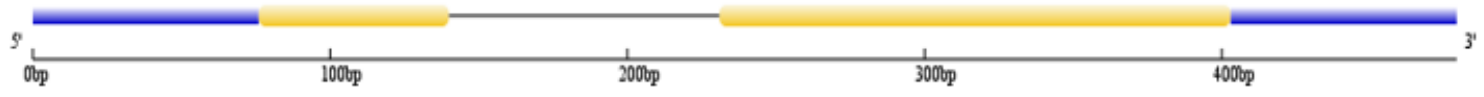

BjuB040111

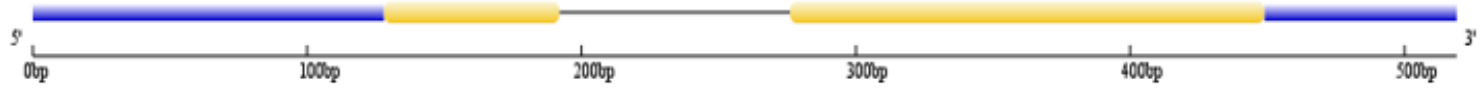

Legend:

CDS $=$ upstrean/downstream - Intron

Figure 5: Gene structure of B. juncea defensin BjuB040113, BjuB040109, BjuB027448, BjuA030410, BjuA025766 and BjuB040111. 
In plants, gene transcription is regulated by cis-acting regulatory elements that bind to target transcription factors. Some cisregulatory elements are involved in stress responses. To analyze how the expression level of $B$. juncea defensins respond to stress stimuli, $2.0 \mathrm{~kb}$ upstream promoter regions of B. juncea defensin genes were scanned for stress-related cis-regulatory elements using the plant CARE online service. Two cis-acting regulatory elements involved in MeJA responsiveness, CGTA-motif and TGACGmotif were identified in the promoter region of $B$. juncea defensin genes denoting to the possible regulation of plant defensin genes under JA responsive pathway. Two light responsive elements GTI and a G-box, TGA-element in auxin responsiveness were identified indicating that these defensin genes might be involved in the response to various light stress and hormone treatments via participating in different regulatory mechanisms. Apart from that, 10 common cis-acting elements in promoter and enhancer region, and 14 core promoter elements around -30 of transcription start site were identified. The type and number of cis-acting regulatory elements in the promoter region of each $B$. juncea defensin gene were discrepant indicating that the members of $B$. juncea defensin gene family might be able to respond to different abiotic stresses. Similar pattern was observed in cis-acting regulatory elements in C. sativa defensin genes.
Phylogenetic analysis of defensin proteins of $B$. juncea, $C$. sativa and related species

A phylogenetic tree was constructed on the basis of full length $B$. juncea defensin protein sequences to understand the phylogenetic relationship among these proteins (Figure 6). According to the MEGA software, the evolutionary history was inferred using the Neighbor-Joining method [43]. The optimal tree with the sum of branch length $=3.45580578$ is shown. The tree is drawn to scale, with branch lengths in the same units as those of the evolutionary distances used to infer the phylogenetic tree. The evolutionary distances were computed using the Poisson correction method [57] and are in the units of the number of amino acid substitutions per site. The proportion of sites where at least one unambiguous base is present in at least one sequence for each descendent clade is shown next to each internal node in the tree. The analysis involved 6 amino acid sequences. All positions containing gaps and missing data were eliminated. There were a total of 77 positions in the final dataset. Evolutionary analyses were conducted in MEGA7. The tree indicates that $B$. junea defensins belong to three distinct groups, majority of defensins clustered under group I. However, the defensin protein sequences of $C$. sativa used in phylogenetic analysis resulting in the categorization into five distinct groups (Figure 7).

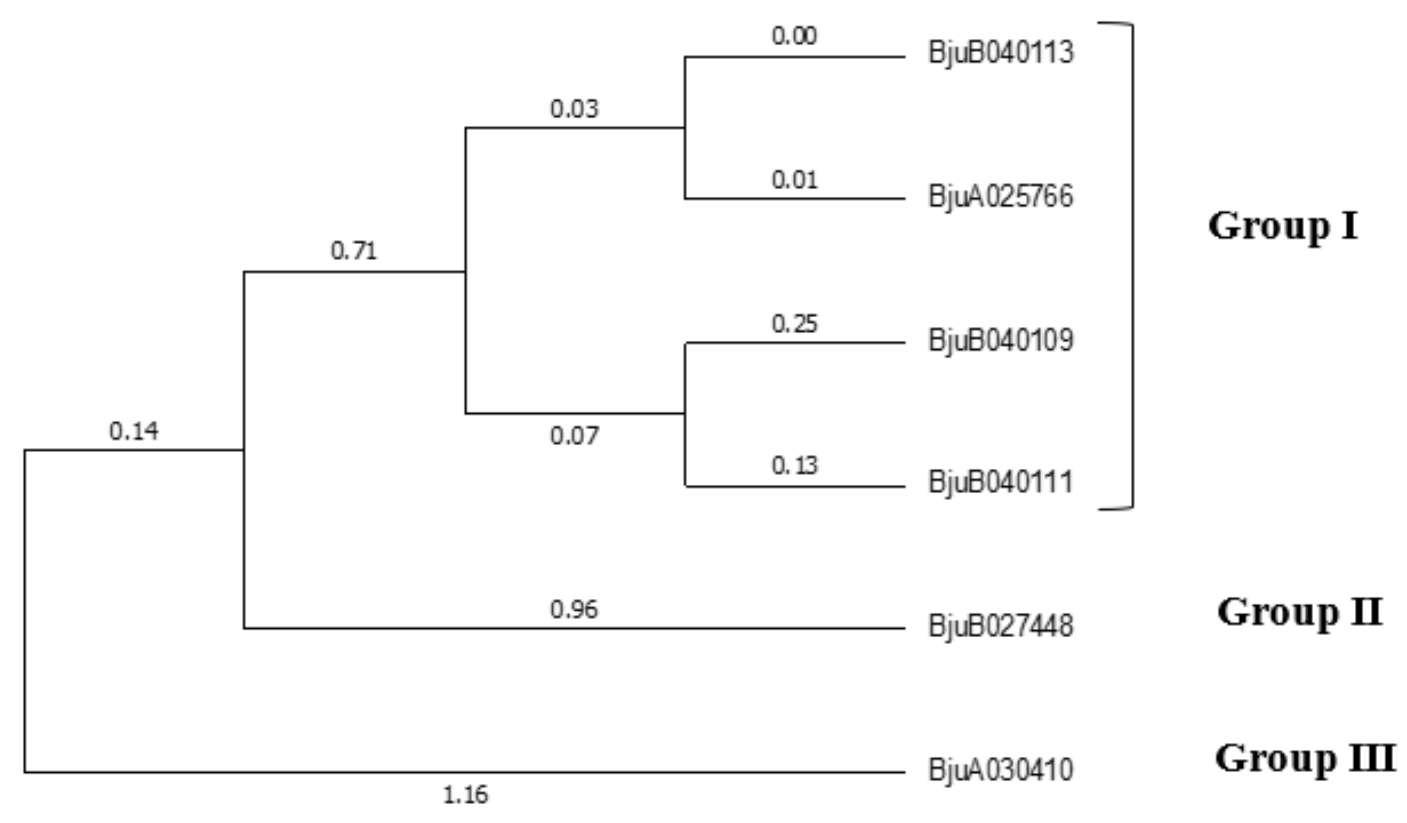

Figure 6: The phylogenetic tree of B. juncea defensin proteins constructed by Neighbor-Joining method. 


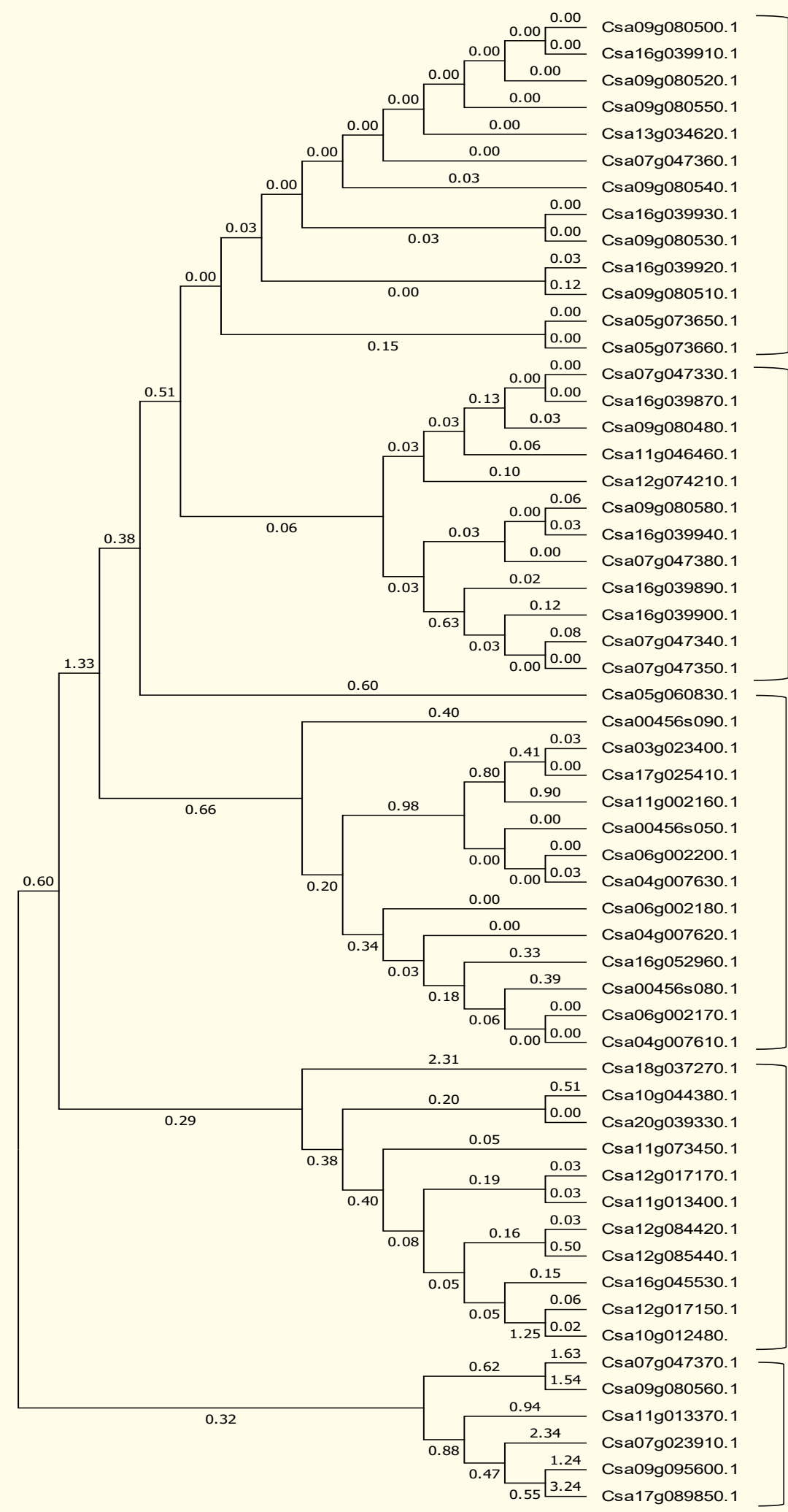

\section{Group I}

\section{Group II}

\section{Group III}

\section{Group IV}

\section{Group V}

Figure 7: The phylogenetic tree of $C$. sativa defensin proteins constructed by Neighbor-Joining method. 
To study the evolutionary relationship of B. juncea and C. sativa to other related species comprehensively, defensin proteins of $B$. napus, B. oleracea, and B. rapa, B. nigra were also obtained from BRAD database. A neighbor-joining tree was constructed with defensin proteins of $B$. juncea and other related species including $B$. napus, B. oleracea, B. rapa and A. thaliana (Figure 8). Phylogenetic analysis showed that all the identified defensins from Brassica spe- cies together with Arabidopsis were clearly divided into five subgroups. Most of the $B$. juncea defensins belong to subgroup I, while one defensin belong to subgroup IV. C. sativa defensins were scattered in every group indicating the evolutionary relationship of $C$ sativa with Brassica species. As also observed by Gachomo., et al. [11], Arabidopsis defensins are spread all over the tree suggesting an ancient divergence.

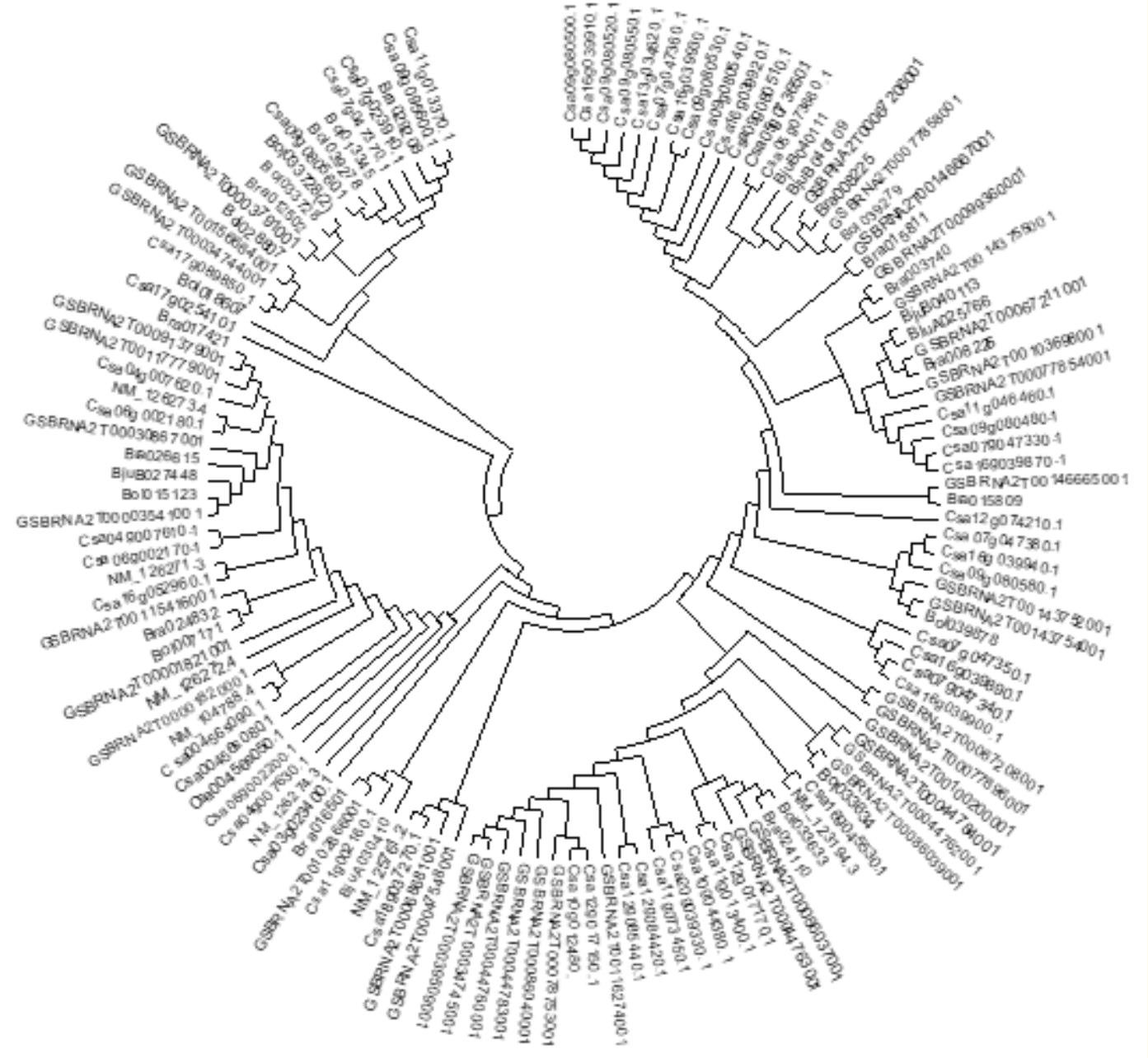

Figure 8: The phylogenetic analysis of B. juncea, B. rapa, B. oleracea, B. napus, C. sativa and

$A$. thaliana defensin proteins by Neighbor-Joining method.

Expression analysis of defensin genes in $B$. juncea and $C$. sativa in response to SA, JA and $A$. brasicae infection and wounding

To study the role of plant defensin in defense of $B$. juncea and C. saiva, induction of defensin genes in response to exogenous application of SA and JA was studied by performing qRT-PCR on RNA isolated from leaf sample at different time intervals. The importance of SA, JA and ET as dominant primary signals in local and systemic induced defense signaling has been well documented. Exogenous application of JA induces a set of defense genes that are also activated upon pathogen infection, among which are genes encod- 
ing plant defensins and thionins [40]. Expression analysis of defensin revealed that both the genotypes respond to JA application. In $B$. juncea the expression of defensin gene increase gradually up to $12 \mathrm{~h}$ and then with a sharp increase, peaked at $48 \mathrm{~h}$ after application. After $48 \mathrm{~h}$, the expression of the gene was declined (Figure $9)$. However, in $C$. sativa the expression was early compared to $B$. juncea, revealing a sharp peak at $6 \mathrm{~h}$ after JA application, and then down-regulated at each time point after treatment. Presence of two cis-acting regulatory elements involved in MeJA responsiveness in promoter region of defensin genes further support the theory of regulation of defensins by JA signaling. Chamil., et al. [4] also reported that upon JA treatment, increased expression of $P R 3$, $P D F 1.2$, and GST was observed. This is in agreement with Arabidopsis defense signaling pathway that JA generally induces PDF1.2 and $P R 3$ and therefore, those two genes have been extensively used as markers for JA signaling pathway [51].

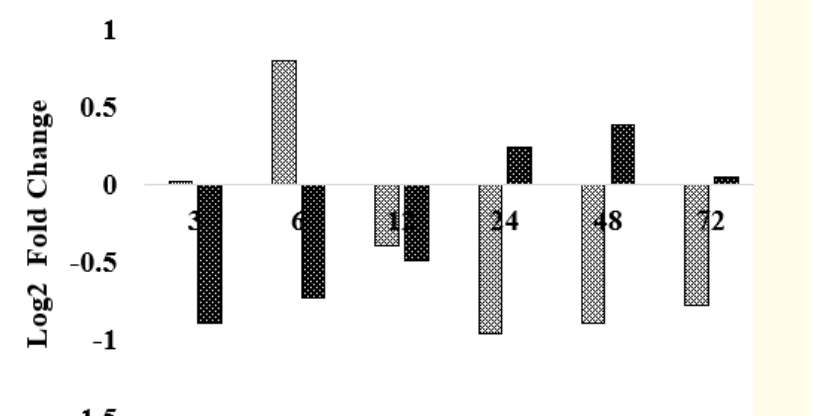

Figure 9: Real-time PCR analysis of defensin genes in $C$. sativa and $B$. juncea in response to exogenous application of JA shows differential expression.

Howver, unlike JA, there was a significantly low level of defensin gene expression in response to exogenous SA application in both the genotypes (Figure 10). The decrease in defensin gene expression by SA coincides with the increased expression by JA confirming the antagonistic interaction between the SA and JA response pathways. Past literature revealed that JA responsive genes such as PDF1.2 are highly sensitive to suppression by exogenous application of SA $[20,40,45]$.

Many studies have demonstrated that endogenously accumulating SA antagonizes JA-dependent defenses, thereby prioritizing SA-dependent resistance over JA-dependent defense. This antagonism between SA and JA signaling was observed in a large number

of Arabidopsis accessions, highlighting the potential significance of SA/JA crosstalk in nature [6]. Though many reports describe SA/ JA antagonism, synergistic interaction have been reported as well [34]. Emerging data suggests that, there is a more complex signaling network evoking both positive and negative regulatory interactions [28]. Furthermore, analysis of some Arabidopsis mutants with constitutive SA responses reveals a pathway in which JA and ET signaling are required for SA responses [54]. Mur., et al. in 2006 reported that in Arabidopsis treatments with low concentrations of JA and SA resulted in a synergistic effect on the JA and SA responsive genes PDF1.2 and PR1, respectively. However, at higher concentrations the effects were antagonistic, demonstrating that the outcome of the SA-JA interaction is dependent on the relative concentration of each hormone. Chamil., et al. [4] also reported that although PDF1.2 is known to be induced by JA but not by SA in Arabidopsis, PDF1.2 could also be induced by SA in $C$. sativa. The JA-responsive genes LOX2, VPS and PDF1.2 contain one or more SA responsive TGACG motifs in their promoters. The occurrence of this motif in defensin gene promoter indicates a cross talk between SA and JA pathways [2].

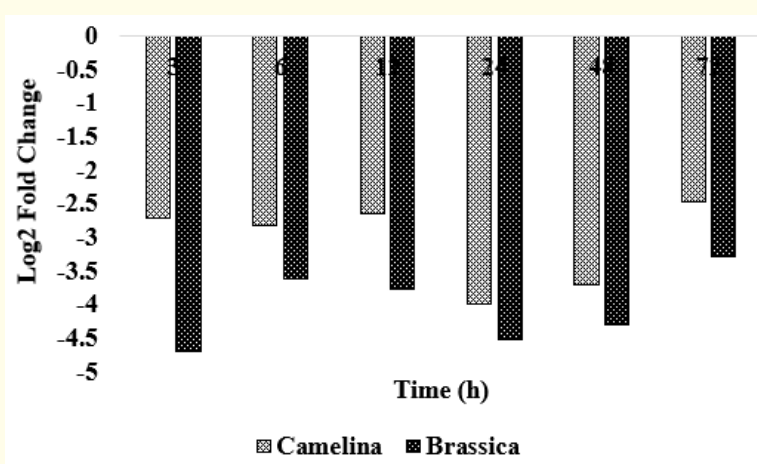

Figure 10: Real-time PCR analysis of defensin genes in C. sativa and B. juncea in response to exogenous application of SA a down-regulation.

In order to study the expression of defensin gene to Alternaria infection, plants were infected with a suspension of $A$. brassicae spores. Quantitative real time PCR analysis indicated that, relative expression of defensin gene in C. sativa in response to Alternaria infection is comparatively very low in spite of the genotypes' resistancy to A. brassicae (Figure 11). The gene induction was very marginal. This can be explained by studying other defensin genes which are many [56] in case the gene in consideration in this study may not involve in resistance. Also might be important are levels of SA and JA in C. sativa which needs to be explained. However, Chamil., et al. [4] demonstrated a remarkable induction of PDF1.2 in locally 
challenged leaves of $C$. sativa in response to $A$. brassicae infection. However, there was a marked increase in defensin gene expression in B. juncea compared to $C$. sativa in response to infection. The relative expression was highest at $24 \mathrm{~h}$, continue till $48 \mathrm{~h}$, and then decreased. According to Pieterse., et al. [40], biotrophic pathogens are generally sensitive to defense responses that are regulated by $\mathrm{SA}$, whereas pathogens with a necrotrophic lifestyle are commonly deterred by defenses that are controlled by JA and ET. An Arabidopsis defensin, activated after necrotrophic fungi invasion, is encoded by PDF1.2 gene and is a predominant marker gene for JA pathway [51]. Since A. brassicae is a necrotrophic pathogen, it is obvious that induction of defensin by the pathogen as defensin gene is in JA signaling pathway.

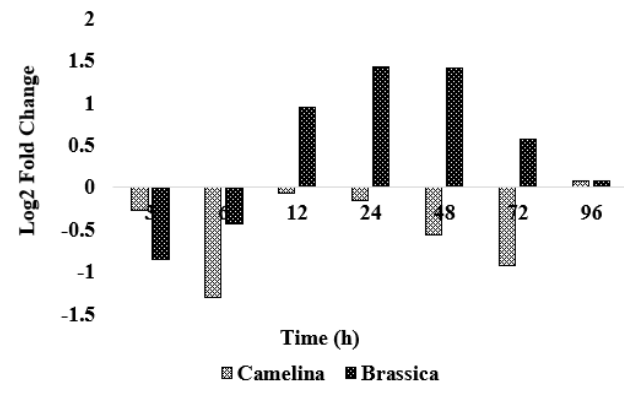

Figure 11: Real-time PCR analysis of defensin genes in $B$. juncea and $C$. sativa in response to a Alternaria infection.

Two genotypes responded differentially to wounding. Expression of defensin gene in wounded $C$. sativa leaves increased initially, showing maximum fold change at $6 \mathrm{~h}$, and then decreased gradually while in $B$. juncea there was a marked decline of the expression from the initial point (Figure 12).

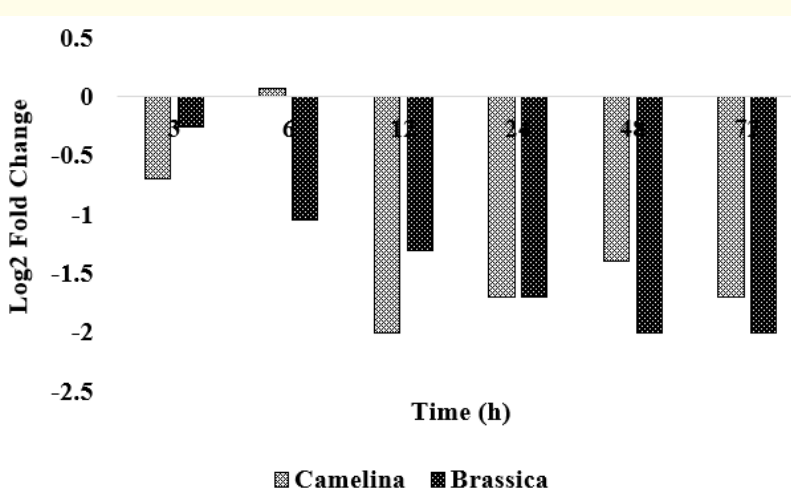

Figure 12: Real-time PCR analysis of defensin genes in B. juncea and C. sativa in response to wounding.
According to Dong [7] the wound induced octadecanoid pathway results in the synthesis of the signal molecule JA, and subsequent activation of genes in defense including defensin. Wounding not only causes a rapid production of JA but the level of ethylene as well [39]. Wounding and JA evoke a very similar response in $C$. sativa in our study further strengthening the idea that wounding exerts its effect through JA signaling. There are two distinct pathways for synthesis of JA. One starts from PLD (phospholipase D) induce by wound, pest, pathogen, elicitors, and the other starts from DAD1 (defective anther dehiscence1) in developmental pathway lead to sysnthesis of AOC (allene oxide cyclase). Growing evidence indicates that developmentally regulated JA biosynthesis in Arabidopsis is controlled through activation of a JA biosynthetic pathway that differs from, but overlaps with the biosynthetic pathway that regulates wound-induced JA biosynthesis [54].

\section{Conclusion}

In-silico analysis and characterization of defensin genes in $B$. juncea and $C$. sativa leads to a better understanding of their roles in response to biotic and abiotic stresses. The wild Camelina poses a significantly higher number of defensin genes compared to Brassica and the proteins were more stable. This may be a possible reason of Camelina's resistance to Alternaria blight. Presence of cis-acting regulatory elements involved in MeJA responsiveness indicates their expression in response to stress conditions. Analysis of defensin gene response to SA, JA, Alternaria infection, and wounding revealed a complex and interesting network of pathways that function both separately and together to render defense responses in both the genotypes. The defensin genes can be used for further studies including antimicrobial studies to find effectiveness of using defensin in transgenic Brassica to confer resistance to $A$. brassicae.

\section{Acknowledgments}

Authors wish to acknowledge the support given by National Phytotron Facility for providing glasshouse facilities. Chaturani GDG acknowledge the receipt of PhD fellowship from Indian Council for Agricultural Research.

\section{Bibliography}

1. Anderson CR., et al. "Transcriptional control of plant defense gene expression". Australian Biochemist 31.4 (2000): 8-11.

2. Anuradha TS., et al. "A defensin gene of Indian mustard is stress induced". Journal of Plant Biochemistry and Biotechnology 8.2 (2009): 221-224. 
3. Carvalho AO and Gomez VM. "Plant defensins - Prospects for the biological functions and biotechnological properties". Peptides 30 (2009): 1007-1020.

4. Chamil NNM., et al. "Defense gene induction in C. sativa upon Alternaria brassicae challenge". Indian Phytopathology 67.3 (2014): 252-256.

5. Colilla FJ., et al. "g-Purothionin: Amino acid sequence of two polypeptides of a new family of thionins from wheat endosperm". FEBS Lettres 270: 191-194.

6. Does DV., et al. "Salicylic acid suppresses jasmonic acid signaling downstream of SCFCOI1-JAZ by targeting GCC promoter motifs via transcription factor ORA59". The Plant Cell 25 (2013): 744-761.

7. Dong X. "SA, JA, ethylene, and disease resistance in plants" Current Opinion in Plant Biology 1 (1998): 316-323.

8. Donnes P and Hoglund. "A Predicting protein subcellular localization: Past present and future". Genome Protein Bioinfomatics 2.4 (2004): 209-245.

9. Ebrahim S., et al. "Pathogenesis related (PR) proteins: chitinase and $\beta$-1,3-glucanase in defense mechanism against malformation in mango (Mangifera indica L.). Scientia Horticulturae 130.4 (2011): 847-852.

10. Epple P., et al. "An Arabidopsis thaliana thionin gene is inducible via a signal transduction pathway different from that for pathogenesis-related proteins". Plant Physiology 109 (1995): 813-820

11. Gachomo EW., et al. "Structural characterization of plant defensing protein superfamily". Molecular Biology Reports 39.4 (2012): 4461-4469.

12. Gasteiger E., et al. "The Proteomics Protocols Handbook, Humana Press". (2005): 571-607.

13. Ghose K., et al. "Deferential profiling of selected defense related genes induced on challenge with Alternaria brassicola in resistant white mustard and their comparative expression pattern in susceptible Indian mustard". Molecular Pant Pathology 9.6 (2008): 763-775.

14. Glazebrook J. "Contrasting mechanisms of defense against biotrophic and necrotrophic pathogens". annual review of phytopathology 43 (2005): 205-227.
15. Hanfrey C., et al. "Leaf senescence in Brassica napus: Expression of genes encoding pathogenesis related proteins". Plant Molecular Biology 30.3 (1996): 597-609.

16. Heyn P., et al. "Introns and gene expression: Cellular constraints, transcriptional regulation, and evolutionary consequences". Bioassays 37 (2015): 148-154.

17. Hoover DM., et al. "Antimicrobial Characterization of Human $\beta$-Defensin 3 Derivatives". Antimicrobial agents Chemother 47 (2003): 2804-2809.

18. Kaewklom S., et al. "Structural and biological features of a novel plant defensin from Brugmansia x Candida". Plos One 13.8 (2018): e0201668.

19. Keller H., et al. "Pathogen induced elicitin production intransgenic tobacco generates a hypersensitive response and nonspecific disease resistance". Plant Cell 11 (1999): 223-235.

20. Koornneef A., et al. "Kinetics of salicylate mediated suppression of jasmonate signaling reveal a role for redox modulation". Panting Physiology 147 (2008): 1358-1368.

21. Kumar D., et al. "Alternaria blight of oilseed Brassicas: A comprehensive review". African Journal of Microbiology Research 8.30 (2014): 2816-2829.

22. Lacerda AF., et al. "Antifungal defensins and their role in plant defense". Front Microbiology 5.116 (2014): 1-10.

23. Lay FT and Anderson MA. "Components of the innate immune system in plants". Current Protein and Peptide Science 6.1 (2005): 85-101.

24. Lay FT., et al. "Isolation and properties of floral defensin from ornamental tobacco and petunia". Plant Physiology 131 (2003): 1283-1293.

25. Leon IP and Montesano M. "Activation of defense mechanisms against pathogens in mosses and flowering plants". International Journal of Molecular Sciences 14.2 (2013): 3178-3200.

26. Liu J and Wang XJ. "An integrative analysis of the effects of auxin on jasmonic acid biosynthesis in Arabidopsis thaliana". Journal of Integrative Plant Biology 48 (2006): 99-103.

27. Liu YJ., et al. "Solution structure of the plant defensin VrD1 from mung bean and its possible role in insecticidal activity against bruchids". Proteins: Structure, Function, and Bioinformatics 63 (2006): 777-786. 
28. Loake G and Grant M. "Salicylic acid in plant defense- the players and protagonists". Current Opinion in Plant Biology 10 (2007): 466-472.

29. Loon LCV and Strien EA. "The families of pathogenesis related protein, their activities, and comparative analysis of PR-1 type proteins". Physiological and Molecular Plant Pathology 55.2 (1999): 85-97.

30. Maarof E., et al. "Cloning and heterogeneous expression of CDef1, a ripening induced defensin in Capsicum annum". Australian Journal of Crop Science 5.3 (2011).

31. Mendez E., et al. "Primary structure and inhibition of protein synthesis in eukaryotic cell free system of a novel thionn, gamma-thionin, from barley endosperm". European Journal of Biochemistry 194 (1990): 533-539.

32. Mishra A., et al. "Molecular cloning and in-silico analysis of functional homologous of hypersensitive response genes induced during pathogenesis of Alternaria Blight in two genotypes of Brassica". journal of proteomics and bioinformatics 3 (2010): 244-248.

33. Moosa A., et al. "Transgenic expression of antifungal pathogenesis-related proteins against phyto-pathogenic fungi- 15 years of success". Israel Journal of Plant Sciences 6 (2017): $122-134$

34. Mur LAJ., et al. "The outcomes of concentration specific interactions between salicylate and jasmonate signaling include synergy, antagonism, and oxidate stress leading to cell death". Plant Physiology 140 (2006): 249-262.

35. Nayanakantha NMC., et al. "Differential expression of defenserelated genes in Synapsis alba and Brassica juncea upon the infection of Alternaria brassicae". Tropical Agricultural Research 27.3 (2016): 123-136.

36. Newman T., et al. "Genes galore: A summary of the methods for accessing the results of large scale partial sequencing of anonymus Arabidopsis thaliana cDNA clones". Plant Physiology 106 (1994): 1241-1255.

37. Nowicki M., et al. "Alternaria black spot of Crucifers: Symptoms, importance of disease, and perspectives of resistance breeding". Transgenic Research 19 (2012): 373-384.

38. Oomen RJFJ., et al. "Plant defensin AhPDF1.1 is not secreted in leaves but it accumulates in intracellular compartments". New Phytologist 192.1 (2011): 140-150.
39. Pieterse CMJ and Loon LC. "Salicylic acid independent plant defense pathways". Trends in Plant Science 4.2 (1999): 52-58.

40. Pieterse CMJ., et al. "Networking by small molecule hormones in plant immunity". Nature Chemical Biology 5.5 (2009): 308316.

41. Rawat S., et al. "Expression analysis of chitinase upon challenge inoculation to Alternaria wounding and defense inducers in Brassica juncea”. Biotechnology Reports 13 (2017): 7279.

42. Sagehashi Y., et al. "Purification and cDNA cloning of a dfensin in Brassica juncea, its functional expression in Escherichia coli, and assessment of its antifungal activity". Pesticide Science 38.1 (2013): 33-38.

43. Saitou N and Nei M. "The neighbor-joining method: a new method for reconstructing phylogenetic trees". Molecular Biology and Evolution 4.4 (1987): 406-425.

44. Saskia CM., et al. "Characterization of the early response of Arabidopsis to Alternaria brassicola infection using expression profiling". Plant Physiology 132 (2003): 606-617.

45. Spoel SH. "NPR1 modulates cross-talk between salicylate and jasmonate dependant defense pathways through a novel function in the cytosol". Plant Cell 15 (2003): 760-770.

46. Sticher L., et al. "Vacular chitinases of tobacco: a new class of hydroxyproline containing proteins". Science 257 (1992): 655657.

47. Stotz HU., et al. "Plant defensins: defense, development and application". Plant Signal Behave 4 (2009): 1010-1012.

48. Tam JP., et al. "Antimicrobial peptides from plants". Pharmaceuticals 8.4 (2015): 711-757.

49. Thevissen K., et al. "A gene encoding a sphingolipid biosynthesis enzyme determines the sensitivity of Saccharomyces cerevisiae to an antifungal plant defensin from dahlia (Dahlia merkii)". Proceedings of the National Academy of Sciences of the United States of America 07 (2000): 9531-9536.

50. Thevissen K., et al. "Interaction of antifungal plant defensins with fungal membrane components". Peptides 24 (2003): 1705-1712.

51. Thomma BPHJ., et al. "Desease development of several fungi on Arabidopsis can be reduced by treatment with methyl jasmonate". Plant Physiology and Biochemistry 38.5 (2000): 421427. 
52. Thomma B., et al. "Mode of action of plant defensins suggests therapeutic potential". Current Drug Targets - Infectious Disorders 3.1 (2003): 1-8.

53. Tiwari S., et al. "Effect of methyl jasmonate on disease severity and expression of plant defensin gene during Alternaria brassicae infection in Arabidopsis". International Journal of Current Microbiology and Applied Sciences 6.7 (2017): 857-865.

54. Turner JG., et al. "The jasmonate signal pathway". The Plat Cell (2002): 153-164.

55. Zhao KJ and Chye ML. "Methyl jasmonate induces expression of a novel Brassica juncea chitinase with two chitin binding domains". Plant Molecular Biology 40.6 (1999): 1009-1018.

56. Zhou P. "Determining protein half-lives. (In): Dickson, R. C. and Mendenhall, M. D. (eds). Signal transduction protocols". Methods in Molecular Biology 284 (2004).

57. Zuckerkandl E and Pauling L. "Evolutionary divergence and convergence in proteins". Evolving Genes and Proteins (1965): 97-166.

Volume 3 Issue 12 December 2019

(C) All rights are reserved by Anita Grover., et al. 